\title{
LOI/SOHO CONSTRAINTS ON OBLIQUE ROTATION OF THE SOLAR CORE
}

\author{
L. GIZON \\ W.W. Hansen Experimental Physics Laboratory, \\ Stanford University, Stanford CA 94305, U.S.A. \\ T. APPOURCHAUX \\ ESA / ESTEC, \\ P.O. Box 299, 2200 AG Noordwijk, The Netherlands \\ AND \\ D.O. GOUGH \\ Institute of Astronomy, and Department of Applied Mathematics \\ and Theoretical Physics, University of Cambridge, \\ Madingley Road, Cambridge CB3 OHA, U.K.; \\ HEPL, Stanford University, Stanford CA 94305, U.S.A.
}

\section{Introduction}

The axis of rotation of the Sun's surface is inclined from the normal to the ecliptic by $7^{\circ} .25$. Is that true also of the rotation of the rest of the Sun? Knowledge of the direction of the angular momentum is pertinent to studies of the formation of the solar system. Moreover, Bai and Sturrock (1993) have recently interpreted temporal variations in the spatial distribution of solar flares as the outcome of the interaction of the Sun's envelope with an obliquely rotating core. We report here an attempt to determine the principal seismic axes of oscillation of the dipole and quadrupole $\mathrm{p}$ modes from LOI data obtained as a component of the VIRGO investigation on the spacecraft SOHO. We find that formally their most likely orientation is somewhat closer to being normal to the ecliptic than is the axis of the surface rotation. However, the uncertainty in the determination well encompasses the possibility of them being parallel to the surface rotation axis, yet it does not reject (at a level marginally greater than one standard deviation) the possibility that the Sun's angular momentum is parallel to that of the rest of the solar system.

\section{The influence of rotation on oscillations}

Rotation splits the degeneracy of oscillation modes in a multiplet (having like radial order $n$ and degree $l$ ). If angular velocity is constant on spheres, a scalar p-mode 
eigenfunction is approximately proportional to $\Re\left[Y_{l}^{m}(\theta, \phi) \exp \left(-\mathrm{i} \omega_{m} t\right)\right]$ with respect to spherical polar coordinates $(r, \theta, \phi)$ about an appropriate axis $\mathbf{P}$. The index $m$ denotes the azimuthal order, and $Y_{l}^{m}$ is a spherical harmonic function. If rotation is about a unique axis $\mathbf{R}$, then $\mathbf{P}$ coincides with $\mathbf{R}$. But if $\mathbf{R}$ varies with $r, \mathbf{P}$ is a vector average of $\mathbf{R}(r)$, weighted with the rotational splitting kernel $K$ of the mode (Gough and Kosovichev, 1993). When the magnitude $\Omega$ of the angular velocity varies with latitude (defined relative to $\mathbf{R}(r)$ ), $K$ depends on $m$ and, except in the case $l=1$, the pulsation axis of the multiplet is not well defined. In the case of the Sun, the latitudinal variation of $\Omega$ is small: in the data analysis of quadrupole modes we report below, we assume that there exists a frame in which each azimuthal component can still be described geometrically in terms of a single spherical harmonic, and that the $m$ dependence of the splitting is negligible. If the oscillation axis $\mathbf{P}$ is not coincident with the surface rotation axis $\mathbf{S}$ with respect to which one imagines solar oscillations are analysed, each harmonic projection $\left(\ell, m^{\prime}\right)$ with respect to $\mathbf{S}$ is actually a linear combination of normal modes, and will exhibit all the rotationally split frequencies $\omega_{m}$ (where $m$ is the true azimuthal order, with respect to $\mathbf{P}$ ). The orientation of $\mathbf{P}$ cannot be determined from frequencies alone, however - the frequencies of an (aspherical) object are independent of the direction from which that object is viewed. To determine $\mathbf{P}$ it is necessary to measure the eigenfunctions. A first attempt at that (Gough, Kosovichev and Toutain, 1995), made by estimating in the IPHIR data the amplitudes of whole-disc projections of blended components of $l=1$ multiplets, suggested that $\mathbf{P}$ might be closer to the normal $\mathbf{n}$ to the ecliptic than is $\mathbf{S}$, but the significance of the measurement is difficult to assess. Here we report a more sophisticated analysis of modes with $l=1$ and $l=2$ observed with the LOI (Appourchaux, Andersen, Fröhlich, et al., 1997).

\section{Determination of the pulsation axes}

The LOI measures radiant intensity $s_{p}(t)$ integrated over a set of 12 pixels $p$. The nominal attitude of SOHO is such that the projection of $\mathbf{S}$ onto the detector should coincide with a principal axis of symmetry $\mathbf{d}$ of the detector at all times. A multiplet $(l, n)$ is presumed to be composed of $2 l+1$ independently randomly excited modes $m$, each producing a disturbance of the form $x_{m}(\theta, \phi, t) \equiv f_{m}\left(t ; \omega_{m}\right) Y_{l}^{m}(\theta, \phi)$ about a pulsation axis $\mathbf{P}$. We denote by $\beta_{d}(t)$ the inclination of the axis $\mathbf{P}$ with respect to $\mathbf{d}$, and by $\alpha_{d}(t)$ the azimuth of $\mathbf{P}$ relative to the line of sight. During the short selected interval of observation, the variation of $\alpha_{d}(t)$ and $\beta_{d}(t)$ is small, and accordingly we ignore it, replacing $\alpha_{d}(t)$ and $\beta_{d}(t)$ by their averages $\bar{\alpha}_{d}$ and $\bar{\beta}_{d}$. This approximation implies that we neglect the hyperfine structure that results from SOHO's orbit about $\mathbf{n}$ (see Goode and Thompson, 1992). The sensitivity of a pixel $p$ to a mode $m$ can thus be expressed in terms of the Euler angles $\bar{\alpha}_{d}$ and $\bar{\beta}_{d}$ by integrating over the pixel the Fourier amplitude $\hat{x}_{m}(\theta, \phi, \omega)$ of $x_{m}$ weighted with the limb-darkening function. Now consider a small frequency range, and construct $2 l+1$ linear combinations $\hat{y}_{M}(\omega)$, $-l \leq M \leq l$, of the 12 Fourier transforms $\hat{s}_{p}(\omega)$ in order to isolate each component $m$. Úsing a maximum likelihood technique, we fit a parametric model to the $2 l+1$ complex spectra $\hat{y}_{M}(\omega)$. For each discrete frequency $\omega, \hat{f}_{m}\left(\omega ; \omega_{m}\right)$ is presumed to be (a realization of) a centred complex Gaussian random variable whose variance is Lorentzian with amplitude $A_{m}$, linewidth $\Gamma$, and central frequency $\omega_{0}+m a_{1}$, where

$a_{1}$ is the mean rotational splitting. The $\hat{f}_{m}$ are assumed to be independent random variables. We add independent Gaussian noise to each pixel with location-dependent 


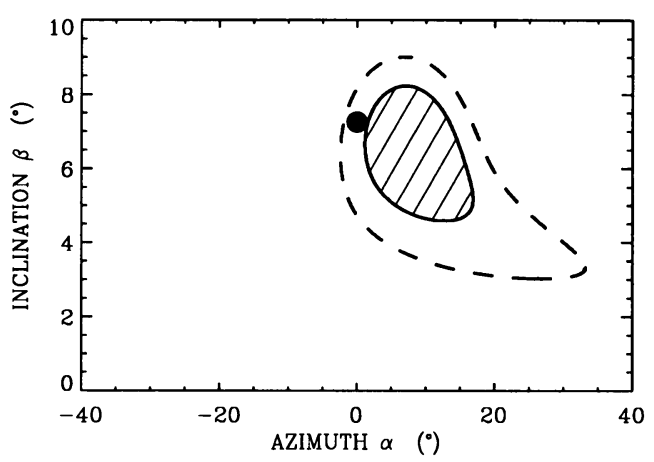

Figure 1. Permitted orientations $(\alpha, \beta)$ of the mean pulsation axis $\mathbf{P}$ with respect to the normal $n$ to the ecliptic. The hatched area and the dashed boundary refer to the 1- $\sigma$ and 1.5- $\sigma$ confidence levels respectively, computed under the assumption that the uncertainties in $\bar{\alpha}_{d}$ and $\bar{\beta}_{d}$ are independent. The filled circle indicates the direction of $\mathbf{S}$.

variance depending on three undetermined parameters $N_{j}$. The parameters $\left(\bar{\alpha}_{d}, \bar{\beta}_{d}\right.$, $\omega_{0}, a_{1}, \Gamma, A_{m}, N_{j}$ ) are chosen such that they maximize the joint probability density function over some frequency interval (frequency bins are assumed to be independent of each other). Realistic Monte Carlo simulations have been performed which demonstrate that the estimators are essentially unbiased.

Observations were taken over the four-month interval 1996, July 10 - Nov. 6 , centred about Sept. 8 when the inclination $B_{0}(t)$ of $\mathbf{S}$ from the axis $\mathbf{d}$ was the greatest (on average, $\bar{B}_{0}=6^{\circ} .06$ ). During this interval the average position of $\mathbf{n}$ is coincident with $\mathbf{d}$. The angles $\bar{\alpha}_{d}$ and $\bar{\beta}_{d}$ where determined for each multiplet separately. Means and standard deviations are listed in Table 1. From the knowledge of the satellite's orbit it is also possible to deduce constraints on the fixed azimuth $\alpha$ and inclination $\beta$ of $\mathbf{P}$ with respect to the ecliptic normal $\mathbf{n}$ (Figure 1). The results are not inconsistent with $\mathbf{P}$ being coincident with $\mathbf{S}$.

TABLE 1. Euler angles $\bar{\alpha}_{d}$ and $\bar{\beta}_{d}$ which define the directions of the pulsation axes $\mathbf{P}$ with respect to $d$.

\begin{tabular}{lll}
\hline mode set & $\bar{\alpha}_{d}$ & $\bar{\beta}_{d}$ \\
\hline$l=1$ and $15 \leq n \leq 24$ & $5^{\circ} \pm 10^{\circ}$ & $4^{\circ} .9 \pm 2^{\circ} .5$ \\
$l=2$ and $14 \leq n \leq 24$ & $10^{\circ} \pm 10^{\circ}$ & $5^{\circ} .7 \pm 1^{\circ} .6$ \\
all & $8^{\circ} \pm 7^{\circ}$ & $5^{\circ} .5 \pm 1^{\circ} .4$ \\
\hline
\end{tabular}

\section{On the orientation of the core}

We adopt a simple two-zone model. The outer zone, $r_{\mathrm{c}}<r \leq R_{\odot}$ is assumed to rotate about $\mathbf{S}$ with angular velocity determined from higher-degree modes (Kosovichev, Schou, Scherrer, et al., 1997); beneath the convection zone a uniform rate of $435 \mathrm{nHz}$ 


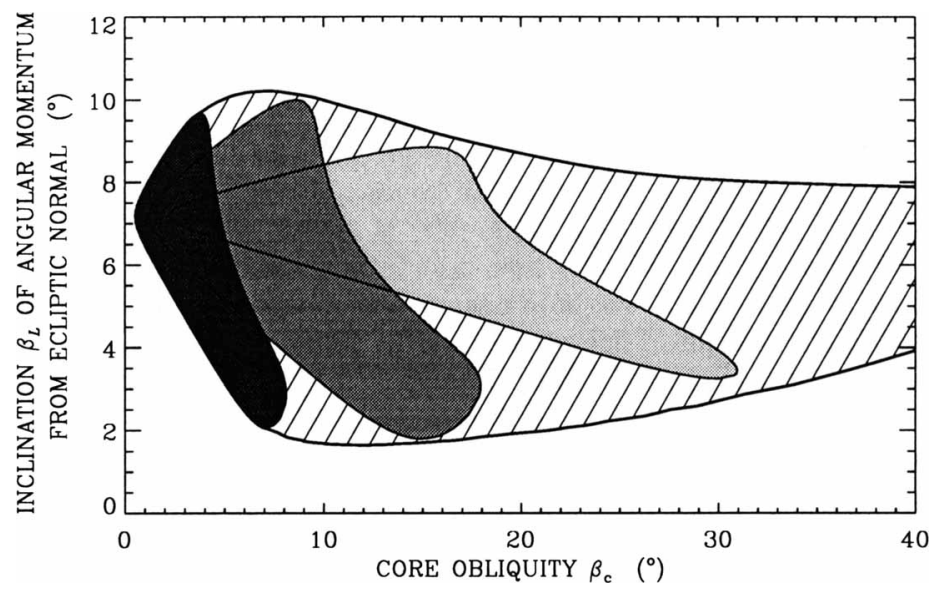

Figure 2. Permitted inclinations $\beta_{L}$ of the solar angular momentum when $a_{1}=440 \mathrm{nHz}$, for $r_{\mathrm{c}} / R_{\odot}=0.25$ (light grey), 0.4 (mid grey) and 0.7 (dark grey). The hatched area is the permitted region for $r_{\mathrm{c}}<0.7 R_{\odot}$ and $433 \mathrm{nHz}<a_{1}<447 \mathrm{nHz}$.

was adopted. The inner zone, $0 \leq r \leq r_{\mathrm{c}}$, was assumed to rotate uniformly with angular velocity $\Omega_{\mathrm{c}}$ about an axis $\mathbf{C}$. The constraints on $(\alpha, \beta)$ can then be used to constrain the obliquity $\beta_{\mathrm{c}}$ of $\mathbf{C}$ with respect to $\mathbf{S}$ for each value of $r_{\mathrm{c}}$. In this way one can test, for example, the model of Bai and Sturrock (1993) in which the core rotates at a rate $\Omega_{\mathrm{c}} / 2 \pi=454 \mathrm{nHz}$ inclined by $\beta_{\mathrm{c}}=40^{\circ}$. In this model, we find that the core radius $r_{\mathrm{c}}$ has to be less than $0.24 R_{\odot}$ in order to be consistent with LOI data.

\section{The orientation of the angular momentum}

Subject to the same two-zone model, we can also constrain the orientation $\left(\alpha_{L}, \beta_{L}\right)$ of the axis $\mathbf{L}$ of the Sun's angular momentum with respect to the normal $\mathbf{n}$. In Figure 2 , the hatched region in the $\left(\beta_{\mathrm{c}}, \beta_{L}\right)$ plane is the permitted region if $\alpha, \beta$ and $a_{1}$ are all within one standard deviation of their seismically determined values. We measured the averaged rotational splitting over the same set of modes to be $a_{1}=440 \pm 7 \mathrm{nHz}$ from one year of data. It is evident that at a level of significance only slightly above one standard deviation the possibility of $\mathbf{L}$ being perpendicular to the plane of the ecliptic is not ruled out.

\section{References}

Appourchaux, T., Andersen, B.N., Fröhlich, C., Jiménez, A., Telljohann, U. and Wehrli, C.: 1997, Solar Phys. 170, 27.

Bai, T. and Sturrock, P.A.: 1993, Astrophys. J. 409, 476.

Goode, P.R. and Thompson, M.J.: 1992, Astrophys. J. 395, 307.

Kosovichev, A.G., Schou, J., Scherrer, P.H., Bogart, R.S., Bush, R., Hoeksema, J.T., et al.: 1997, Solar Phys. 170, 43.

Gough, D.O. and Kosovichev, A.G.: 1993, in Weiss, W.W. and Baglin, A. (eds.), Inside the Stars, ASP Conf. Series 40, pp. 566-568.

Gough, D.O., Kosovichev, A.G. and Toutain, T.: 1995, in Ulrich, R.K., Rhodes, E.J. Jr. and Däppen, W. (eds.), GONG'94, ASP Conf. Series 76, pp. 55-58. 